\title{
IOT Based LORA Technology for Precision Agriculture
}

Hiba Iftikhar ${ }^{*}$, Ranjit Biswas, Nashrah

Department of Computer Science Engineering Jamia Hamdard University, Delhi, India

DOI: $10.36348 /$ sjet.2020.v05i04.004

| Received: 17.03.2020 | Accepted: 24.03.2020 | Published: 21.04 .2020

*Corresponding author: Hiba Iftikhar

Abstract

Agriculture is the backbone of the economic system declining its production due to overpopulation and urbanization, so there is a need of precision agriculture. Precision agriculture requires sensor integration, automatic control, and networking and data processing capabilities. With the implementation of rapidly growing IoT, challenges of based sitespecific farming practice with the aim of maximizing the crop production along with the efficient usage of resources involved in farming process. It provides real time assessment of various crop and environment parameters by précising the observed farming land as well as the amount of required water, fertilizer and other input needs. This method enables our farmers to achieve efficiency with proper amount in the required time and therefore ensures growth of non-toxic safe and healthy crop. LPWAN is a wireless network technology, which can be inculcated in the agriculture farms for precision purpose. In this study Lora based technology is used to monitor the agriculture parameters i.e; temperature, humidity and soil moisture to realize timely monitoring of the crop growth parameters, which solves the problem of manually collecting the data and overcomes the problem of long distance communication and anti-interference of the intelligent systems. The hardware installation of reducing the sensor implementation is also represented in the study.

Keywords: IOT Based LORA Precision Agriculture.

Copyright @ 2020: This is an open-access article distributed under the terms of the Creative Commons Attribution license which permits unrestricted use, distribution, and reproduction in any medium for non-commercial use (NonCommercial, or CC-BY-NC) provided the original author and source are credited.

\section{INTRODUCTION}

Agriculture is the backbone of the economic system declining its production due to overpopulation and urbanization, so there is a need of precision agriculture. Precision agriculture requires sensor integration, automatic control, and networking and data processing capabilities. With the implementation of rapidly growing IoT, challenges of based site-specific farming practice with the aim of maximizing the crop production along with the efficient usage of resources involved in farming process. It provides real time assessment of various crop and environment parameters by précising the observed farming land as well as the amount of required water, fertilizer and other input needs. This method enables our farmers to achieve efficiency with proper amount in the required time and therefore ensures growth of non-toxic safe and healthy crop. LPWAN is a wireless network technology, which can be inculcated in the agriculture farms for precision purpose. In this study Lora based technology is used to monitor the agriculture parameters i.e; temperature, humidity and soil moisture to realize timely monitoring of the crop growth parameters, which solves the problem of manually collecting the data and overcomes the problem of long distance communication and anti- interference of the intelligent systems. The hardware installation of reducing the sensor implementation is also represented in the study.

Agriculture is the basic level of the people in India. The crop development is needed as that of population explosion in the world. The factors responsible for deteriorating condition of the crop development in the field of agriculture may be due to water wastage, low soil fertility, climate changes etc. There is a need for the optimization of the agricultural resources by the integration of IOT wireless sensor network[1]. The IOT can be defined, as the physical things of the world can be computer if connected to internet. The LPWAN (low power wide area network) as a wireless network protocol operates in sub-GHZspectrum that operates in free of cost, which makes the system cost effective. Lora has the benefit of extended transmission range, extended long battery life. Lora gateway is used to receive the data from the nodes, which can be mobile or static. Lora provides transmission parameters, transmission power (transmit signal can be set between $4 \mathrm{dBm}$ to $20 \mathrm{dBm}$, higher TP increases immunity from the interference), carrier frequency (value between 137MHZ to 1020MHZ), spreading factor (higher spreading factor higher the 
Hiba Iftikhar et al., Saudi J Eng Technol, April, 2020; 5(4): 150-155

range, higher the SNR and higher power consumption). Lora modification features include. Scalable bandwidth, high robustness, low cost, low power, enhanced network capability, long-range link, fading resistance and Doppler resistance. The Lora technology is used as it can fetch the data from the sensor nodes to the longrange distance to the gateway nodes consuming very less power as compared to other wireless network technology.

\section{Types of wireless network used for precision} purpose

1. ZigBee technology: The network technology of the ZigBee wireless has a range of 50 to $100 \mathrm{~m}$ operating at a frequency of $868 \mathrm{MHZ}$. The power consumption and the complexity is low. It operates on AES encryption technique of 128 bits.

2. Bluetooth/BLE technology: This technology has a range of 10 to $100 \mathrm{mts}$ operating at a frequency of 2.4. The power consumption is medium and the complexity is high.

3. Wi-Fi technology: This technology has a range of 50 to $100 \mathrm{mts}$ operating at a frequency of 2.4 and 5GHZ.The power consumption and the complexity is high.

4. Lora technology: This technology has range of 15 to $30 \mathrm{miles} / \mathrm{up}$ to $25 \mathrm{Kms}$ operating in license free sub-GHZ frequency 433 MHZ. The Lora technology consumes less power can elevate the battery lifetime of 20years, supports high capacity i.e; can add millions of messages per base station, enables interoperability. The use of Lora technology can lead to cost effective installation.

\section{Advantages of Lora}

1. Limiting the wastage of resources by optimizing the resources with time and quantity.

2. Removes the headache of the farmers by continuously accessing there farm lands by applying smart farming in agriculture.

3. Offers better and accurate results by planning the inherent variability of farming conditions.

4. Lora source lost for many years because of less power consumption.

5. The maintenance cost is less due to long range and low power consumption.

6. Lora physical layer utilizes ISM bands of 868 and 915HZ- frequency is free to use anywhere.

7. The network capacity for Lora is high.

8. Lora technology is easy to deploy.

\section{Strategies of Lora technology parameters}

There are three main types of parameters to consider

1. Spreading Factor (SF): The Lora technology should have higher spreading factor as the spreading factor means higher range. The symbol for the spreading factors spread by the spreading code of length $2^{\text {sf }}$ chips.

2. Coding Rate (CR): The CR can be described as the number of bits that the receiver can use to detect the payload of the lora packet.

3. Bandwidth (BW): Lora provides the bandwidth frequency in $125 \mathrm{KHZ}, 250 \mathrm{KHZ}$ and $500 \mathrm{KHZ} \mathrm{BW}$ describes how much data can be sent over a particular connection in a given amount of time.

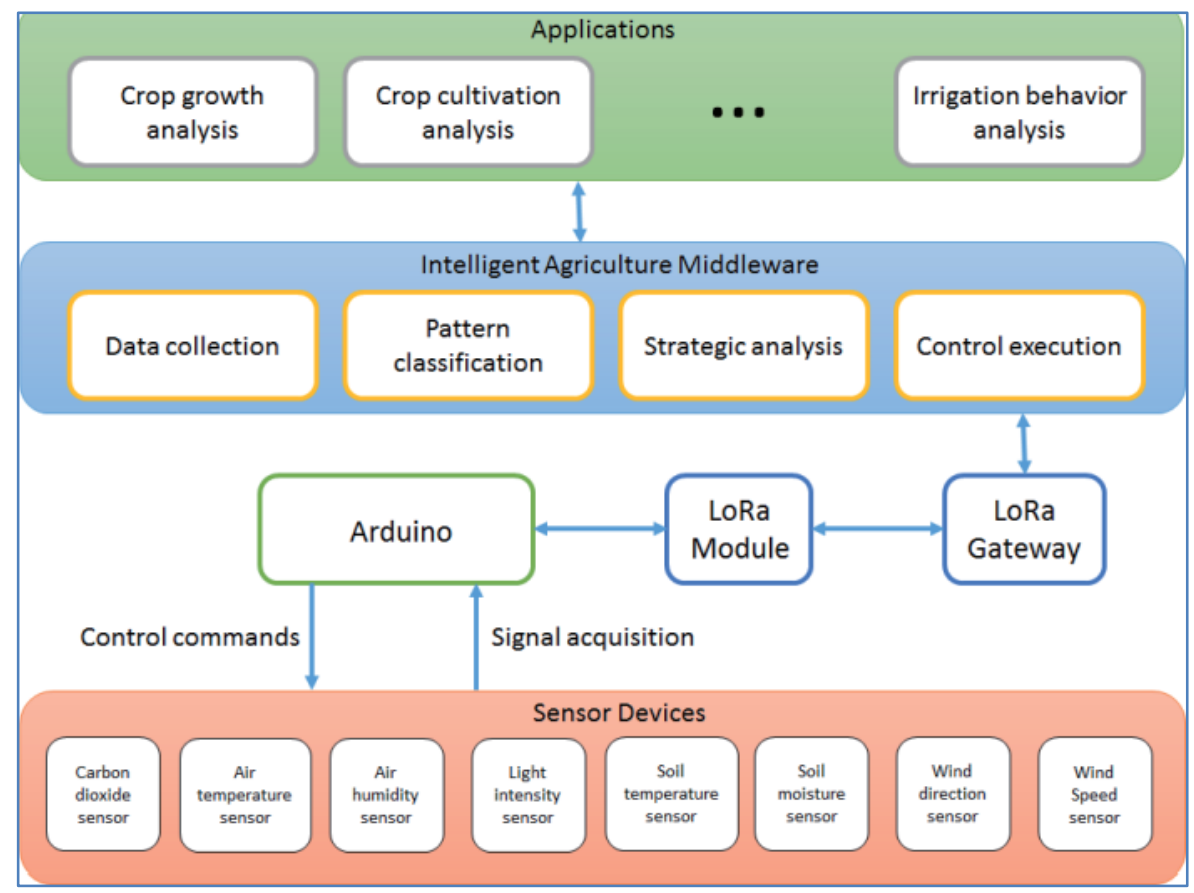

Fig-1.1: Overall system architecture for smart farming 
Hiba Iftikhar et al., Saudi J Eng Technol, April, 2020; 5(4): 150-155

Table-1.1: Comparison of different wireless technologies [3]

\begin{tabular}{|l|l|l|l|l|l|}
\hline & Tx Range & Tx Rate & Tx Power & $\begin{array}{c}\text { Sleep } \\
\text { Power }\end{array}$ & cost \\
\hline Bluetooth(FBT06) & $15 \mathrm{~m}$ & $3 \mathrm{Mbps}$ & $20 \mathrm{~mA}$ & $16 \mathrm{uA}$ & low \\
\hline LoRa(SX1278) & $3000 \mathrm{~m}$ & $2.4 \mathrm{Kpbs}$ & $110 \mathrm{~mA}$ & $2.0 \mathrm{uA}$ & low \\
\hline 3G/4G(U8300) & & $14 \mathrm{Mbps}$ & $800 \mathrm{~mA}$ & $50 \mathrm{uA}$ & high \\
\hline ZigBee(REX3DP) & $100-200 \mathrm{~m}$ & $250 \mathrm{Kbps}$ & $200 \mathrm{~mA}$ & $0.4 \mathrm{uA}$ & low \\
\hline Wi-Fi(CC3200) & $150 \mathrm{~m}$ & $3 \mathrm{Mbps}$ & $75 \mathrm{~mA}$ & $3.5 \mathrm{~mA}$ & high \\
\hline
\end{tabular}

\section{LITERATURE REVIEW}

Many Researchers have worked for smart farming techniques to get the maximum yield by limiting the wastage of resources to have optimization of resources in terms of quantity and time. A detailed review of previously done research is presented below.

JingLiu et al. [2] proposed the intelligent system that overcomes the traditional way of collecting and sensing the parameters of environment of greenhouse crops by using the LORA technology and Iot technology to solve low collection efficiency and cost inefficiency through long-distance communication with strong anti-interference capability and low power consumption required by the current smart farming system.

Sarkar. K.V et al. [3] unpredicted latency to perform sensitive applications. The study is about the Lora technology to decrease the latency of edge nodes to perform edge computing for reduction ao amount of load, amount of unprocessed data the study is encrypted by AES- 128 at 8 - bit AVR Network.

Suresh. M Vignesh et al. [4] proposed the study of machine learning applied on the edge devices to save the bandwidth and power transfer. The end devices capture the raw data and perform data classification before fed to gateway after that to the network server, application servers and end users. The $\mathrm{KNN}$ algorithm is mainly used for classification due to its ease and simplicity of implementation and high sufficiently high accuracy of 96.2 per cent for usage, compressing the transmitted data by 512 times and increasing the battery life by three times.

Adriano, D Jos'e et al. [5] proposed the study of the work of sensor mote that incorporates the sensors for integration of soil and microclimate with the agriculture. The recommendation meets the criteria of low complexity and low cost. The four sensors are proposed two moisture sensors based on capacitance measurement and humidity sensor.

Ikhsan. GMukhammad et al. [6] proposed the study of extended range of transmission, extended long battery life by Lora technology. Lora gateway is used to receive the data from the nodes which can be mobile or static .Loraprovides transmission parameters, transmission power (signal transmission between 4 $\mathrm{dBm}$ to $20 \mathrm{dBm}$, higher TP increases immunityfrom the interference),carrier frequency(value between $137 \mathrm{MHZ}$ to $1020 \mathrm{MHZ}$ ), spreading factor(higher spreading factor higher the range, higher the SNR and higher power consumption).in this paper Lora gateway is used to monitor the health condition of cows measuring the blood pressure, body temperature and heartbeat. The Lora technology is used as it has different functionalities a geolocation i.e this function allows us to detect the location of existing without charge, low cost in terms of infrastructure and operational sensors. Lorasim simulation is used to find the best solution, has a great effect on the increase in DER (data extraction rate) and decrease in NEC (Network energy consumption) of nodes per gateway. This paper compares the result of using static single or multiple gateways to the mobile gateway. The comparison is on the basis of DER and NEC. The one static gateway is preferred over one mobile gateway in terms of DER and NEC if the mobile gateway stops after only one track. The multiple gateways have high DER but energy consumption is very high. Thus the one mobile gate way is preferred if it crosses more than two tracks as it has high data rate and low energy consumption rate than one or multiple static gateways.

Islam Ariful et al. [2]provides the study of automation of the irrigation agricultural land by implementing sensor nodes, gateway nodes, irrigation nodes, database, web application and android application. The irrigation node consists LoRasx1278 with antenna, Arduino Nano, motor control circuit and $12 \mathrm{v}$ voltage solar panel for efficient utilization of water in the agricultural fields.

Kodali. K Ravi et al. [7]studied the sensored data is temperature and humidity for real time monitoring of field, weather prediction by using predictive algorithms utilizing transmitter node:DHT11, soil moisture sensor, ESP3-Lora device, receiver node: ESP-LORA device, IBM Cloud. The high productivityis gained by using the Lora wan protocol maintaining the resources the efficient manner.

Rachmani. F Achmad et al. [8] proposed the study of design of IOT for starfruit by implanting the Lora technology for attaining the maximum range. The average value of the RSSI value, SNR and PDR value is calculated and to get the average time response value. 
Hiba Iftikhar et al., Saudi J Eng Technol, April, 2020; 5(4): 150-155

Ramos. J P et al. [9] proposed the study of SVM algorithm to classify the 42 color features of coffee in which the accuracy for the ripe $=82.54-87.83$ $\%$, semi ripe $=68.25-85.36 \%$, unripe $=76.91-81.39 \%$.

Pantazi. E. X et al. [10] proposed the study of the ANN (artificial neural network) to get the normalized value of wheat prediction with accuracy $81.65 \%$

Senthilnath $\mathrm{J}$ et al. [11]studies the observation of the high spatial resolution of tomato through RGB imaging technology using clustering algorithm.

Zhang Chu et al. [12] proposes the study of observation of the foreign matter in the cotton by shortwave hyper spectral images for classification of botanical and non-botanical foreign matter by SVM algorithm and getting the accuracy of $95 \%$.

NahviBehnaz et al. [13] proposes the calculation of soil temperature on the basis of the weather condition, average air temperature and atmospheric humidity. The estimation of the soil temperature of 6 different depths i.e; $5 \mathrm{~cm}$ $, 10 \mathrm{~cm}, 20 \mathrm{~cm}, 50 \mathrm{~cm}$ and $100 \mathrm{~cm}$ is done by using ANN algorithm having accuracy $87 \%$.

Reshma .J and. Pillai A et al. proposed the study stating how Machine Learning and IoT plays an important role in the field of agriculture. This paper states that most of the work related to soil prediction is based on supervised learning techniques. SVM and ANN techniques are used for soil prediction.

U. Shruthi et al. [14]provides a study claiming that digital image processing can be used to identify the infected area's diseased leaves, roots, fruit and flowers, shape and colour. Image Acquisition, Annoted Dataset, Image processing, Feature extraction, classification is the five stages that the algorithm should follow. Different classifiers are applied on different type of crops and the accuracy of acceptance was determined. SVM, KNN, CNN, Fuzzy are some of the classifiers. Out of them, CNN gives the highest accuracy.

Goldstein. A et al. [15] provides the study that conveys that the data from the sensors is sometimes noisy. In order to use the data by avoiding the noise the author proposes that considering the moisture values smaller than 17 or higher than $39 \%$, are abnormal, and were filtered out. Performance of the simple linear regression models for the different datasets is calculated and shown in the table. Linear regression and two nonparametric approaches, GBRT and BTC are used to determine the models. The models were trained against eight different feature subsets and the result or the accuracy is shown.
Chlingaryan. et al. [16] proposes a study concluding the rapid progress of sensing technologies and ML techniques to provide cost-effective and comprehensive solutions for better estimation and decision-making of crops and the environment. RSbased strategies require enormous quantities of remotely sensed data from different platforms to be processed and hence greater attention is currently being paid to machine learning (ML) methods. This paper addresses scientific advances over the past 15 years on machine-based learning techniques for precise crop yield prediction and estimation of nitrogen status.

Rahman. Z et al. [17] proposed a model that can forecast land type soil sequence and recommend suitable crops as predicted. For soil classification, various machine learning algorithms such as k-NN, Bagged Trees and kernel-based SVM are used. Many datasets has been filtered and the labels which are necessary for the classification are extracted. The results that they get show that SVM perform good accuracy when compared to $k$-NN and Bagged Trees. Providing fertilizer recommendation is the future concern of the researcher.

Popovic .T et al. [18] proposes the study that deals how to monitor the field by using various methods like node system, drone imaging of the crop etc. The data can be sent through the cloud or satellite to the remote users. This paper describes a case study of architecture derivation for a private IoT-enabled system for precision farming, aquaculture, and ecological monitoring research.

Math M. RajinderKumar and Dharwadkar .V Nagaraj et al. [19] proposes the study that focuses on developing a mini weather station to check the climatic conditions by monitoring them and taking necessary actions accordingly. The data is sent to the farmers in real-time and thereby they will take care about the things required for precision agriculture. ZigBee protocol and Arduino are used to monitor and send the information to target device. The future scope of this paper is to add more sensors used to detect wind speed and direction.

Prathibha S R1 et al. [21] explains how IoT helps in smart agriculture. Technologies like sensors and image processing devices are used in this paper. The camera will capture the photos and send to the farmer via MMS through GSM module. This paper will help us in the future if there is scarcity or nonavailability of internet in some villages or at the places where the network coverage is poor.

Jaiganesh. S, Gunaseelan. $\mathrm{K}$ et al. [20]proposed the study of Internet of things (IOT) in Agricultural Sector. This paper deals with shrewd farming. This means applying big data to IoT which interacts with the general population, and in the 
Hiba Iftikhar et al., Saudi J Eng Technol, April, 2020; 5(4): 150-155

evidentiary view, decisions can be made. The author discusses the advantages of IoT in agriculture by reducing cost of generation, protection of the earth. This paper deals with how big data, cloud computing along with IoT shows impact in the field of agriculture.

\section{Scope of Study}

The use of wireless network has been used in many fields as of:

- Flood detection.

- Smart grid.

- Home automation

- Vehicle flow monitoring.

The use of Lora wireless technology has been to produce healthy and productive ranches by predicting the beginning of disease and by determining improved nutrition, automating irrigation, detecting foreign matter like weeds in crops.

This study focuses on wireless network technology based in Lora in the agricultural field, since agriculture is the primary source of population survival. Lora based wireless technology is used as it is cost effective used for long transmission utilizes less power to sense the parameters like temperature, humidity and moisture to increase the yield by providing the accurate information to the farmers.

\section{CONCLUSION}

WSN (wireless network technologies) are used for smart monitoring due to low cost and simple end devices in the field. There are many choices for the communication protocols but Lora comes on the top as a robust, simplistic, low-cost and highly flexible solution. Lo Ra WAN determines the network's communication protocol and device architecture while the physical layer of Lora enables long-range communication connections. The protocol and network architecture have the greatest influence in determining a node's battery life, network capacity, service quality, safety, and the variety of network-serving applications. A network with limited infrastructure can be installed, and as capacity is required, more gateways can be added, data rates changed, the amount of overhearing to other gateways decreased, and the bandwidth scaled. The offer of LPWAN technologies provides a comparison with other protocols:

- Flexibility to target a wide range of applications

- Is the protocol of communication secure?

- Technical aspects - distance, efficiency, interference robustness, two-way communication

- Deployment cost, end-node cost of BOM, battery cost (largest contributor to BOM) Ecosystem of solutions providers for flexible business models.

- Availability of end-products to ensure ROI of network deployment.
- End-product availability to ensure network implementation ROI.

- Ecosystem power to ensure the solution's performance and durability.

The thesis focused on the design, development and applications of Lora technology using low cost solution, long range transmission and low power consumption.

\section{REFERENCE}

1. Anusha, A., Guptha, A., G.(2019). Sivanageswar Rao, and R. K. Tenali, "A model for smart agriculture using IOT," Int. J. Innov. Technol. Explor. Eng., 8(6), 1656-1659.

2. Islam, A., Akter, K., Nipu, N. J., Das, A., M.(2018). Mahbubur Rahman, and M. Rahman, "IoT Based Power Efficient Agro Field Monitoring and Irrigation Control System: An Empirical Implementation in Precision Agriculture," 2018 Int. Conf. Innov. Sci. Eng. Technol., no. October, 372377, 2019.

3. Sarker, V. K., \& J. P.E. (2019). “A Survey on LoRa for IoT: Integrating Edge Computing," 295 300 .

4. Suresh, V. M., Sidhu, R., Karkare, P., Patil, A., Lei, Z., \& Basu, A. (2018, February). Powering the IoT through embedded machine learning and LoRa. In 2018 IEEE 4th World Forum on Internet of Things (WF-IoT) (pp. 349-354). IEEE.

5. Adriano, J. D., Mendes, Y. C., Marcondes, G. A., Furtado, V., \& Rodrigues, J. J. (2018, October). An IoT Sensor Mote for Precision Agriculture with Several MAC Layer Protocols Support. In 2018 International Conference on Information and Communication Technology Convergence (ICTC) (pp. 684-688). IEEE.

6. Ikhsan, M. G., Saputro, M. Y. A., Arji, D. A., Harwahyu, R., \& Sari, R. F. (2018, November). Mobile LoRa Gateway for Smart Livestock Monitoring System. In 2018 IEEE International Conference on Internet of Things and Intelligence System (IOTAIS) (pp. 46-51). IEEE.

7. Kodali, R. K., Yerroju, S., \& Sahu, S. Smart Farm Monitoring Using LoRa Enabled IoT.

8. Rachmani, A. F., \& Zulkifli, F. Y. (2018, October). Design of iot monitoring system based on lora technology for starfruit plantation. In TENCON 2018-2018 IEEE Region 10 Conference (pp. 12411245). IEEE.

9. Ramos, P. J., Prieto, F. A., Montoya, E. C., \& Oliveros, C. E. (2017). Automatic fruit count on coffee branches using computer vision. Computers and Electronics in Agriculture, 137, 9-22.

10. Pantazi, X. E., Moshou, D., Alexandridis, T., Whetton, R. L., \& Mouazen, A. M. (2016). Wheat yield prediction using machine learning and advanced sensing techniques. Computers and Electronics in Agriculture, 121, 57-65.

11. Senthilnath, J., Dokania, A., Kandukuri, M., 
Ramesh, K. N., Anand, G., \& Omkar, S. N. (2016). Detection of tomatoes using spectral-spatial methods in remotely sensed RGB images captured by UAV. Biosystems engineering, 146, 16-32.

12. Zhang, C., Liu, F., \& He, Y. (2018). Identification of coffee bean varieties using hyperspectral imaging: Influence of preprocessing methods and pixel-wise spectra analysis. Scientific reports, $8(1)$, $1-11$.

13. Nahvi, B., Habibi, J., Mohammadi, K., Shamshirband, S., \& Al Razgan, O. S. (2016). Using self-adaptive evolutionary algorithm to improve the performance of an extreme learning machine for estimating soil temperature. Computers and Electronics in Agriculture, 124, 150-160.

14. Shruthi, U., Nagaveni, V., \& Raghavendra, B. K. (2019, March). A review on machine learning classification techniques for plant disease detection. In 2019 5th International Conference on Advanced Computing \& Communication Systems (ICACCS) (pp. 281-284). IEEE.
15. Goldstein, A., Fink, L., Meitin, A., Bohadana, S., Lutenberg, O., \& Ravid, G. (2018). Applying machine learning on sensor data for irrigation recommendations: revealing the agronomist's tacit knowledge. Precision agriculture, 19(3), 421-444.

16. Chlingaryan, A., Sukkarieh, S., \& Whelan, B. (2018). Machine learning approaches for crop yield prediction and nitrogen status estimation in precision agriculture: A review. Computers and electronics in agriculture, 151, 61-69.

17. Rahman, S. A. Z., Mitra, K. C., \& Islam, S. M. (2018, December). Soil classification using machine learning methods and crop suggestion based on soil series. In 2018 21st International Conference of Computer and Information Technology (ICCIT) (pp. 1-4). IEEE.

18. Popović, T., Latinović, N., Pešić, A., Zečević, Ž., Krstajić, B., \& Djukanović, S. (2017). Architecting an IoT-enabled platform for precision agriculture and ecological monitoring: A case study. Computers and electronics in agriculture, 140, 255-265. 